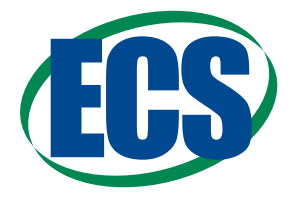

JeS Focus Issue of Selected Papers from IMLB 2018

\title{
Electrochemically Controlled Deposition of Ultrathin Polymer Electrolyte on Complex Microbattery Electrode Architectures
}

\author{
Muhammad E. Abdelhamid, ${ }^{1, z}$ Thomas Rüther, ${ }^{2, z}$ Jean-Pierre Veder, ${ }^{1,3}$ Theo Rodopoulos, ${ }^{1}$ \\ Anand I. Bhatt, ${ }^{2}$ Junqiao Lee, ${ }^{3}$ Anthony F. Hollenkamp, ${ }^{2}$ Mike D. Horne, ${ }^{1}$ Thuy Huynh, ${ }^{2}$ \\ Andojo Ong, ${ }^{4}$ Kate J. Putman, ${ }^{3}$ Genna Rowe, ${ }^{2}$ and Paulo de Souza ${ }^{4}$ \\ ${ }^{1}$ CSIRO Mineral Resources, Clayton VIC 3168, Australia \\ ${ }^{2}$ CSIRO Energy, Clayton VIC 3168, Australia \\ ${ }^{3}$ Curtin University, Bentley WA 6845, Australia \\ ${ }^{4}$ CSIRO Data61, Sandy Bay TAS 7005, Australia
}

Solid state microbatteries are highly sought after for emerging microsensor technologies. To overcome the problem of the dwarfing capacity resulting from the miniaturization of the battery, 3D-structured platform consisting of high surface area micropillar-shaped electrodes are used. However, applying a conformal and continuous solid polymer electrolyte films onto the intricate 3D electrodes is a crucial step toward achieving functional microbatteries. In this work, we present our approach for the development of polyethylene oxide (PEO)-acrylate based ion conducting polymer thin films which function as solid polymer electrolyte (SPE) and a separator. The SPEs were electrochemically deposited on the 3D electrodes resulting in ultrathin, continuous, conformal, and pinhole-free polymer films. The electrochemical and $\mathrm{Li}^{+}$ions transport properties of the SPEs were characterized by EIS measurements and cyclic voltammetry. Furthermore, the homogenous composition of the SPEs at various depths were confirmed by XPS depth profiling techniques.

(C) The Author(s) 2019. Published by ECS. This is an open access article distributed under the terms of the Creative Commons Attribution 4.0 License (CC BY, http://creativecommons.org/licenses/by/4.0/), which permits unrestricted reuse of the work in any medium, provided the original work is properly cited. [DOI: 10.1149/2.0601903jes]

(cc) BY

Manuscript submitted October 26, 2018; revised manuscript received January 11, 2019. Published January 30, 2019. This paper is part of the JES Focus Issue of Selected Papers from IMLB 2018.

Solid and gel polymer electrolytes (SPEs) have received considerable attention in energy storage devices because they offer significant advantages over liquid electrolytes. These include enhanced safety due to the elimination of flammable solvents and reduced engineering efforts (and hence cost) due to the elimination of a costly separator and electrolyte leakage for example. ${ }^{1-4}$ The improved safety aspect due to avoidance of toxic and liquid electrolytes is even more important when implantable medical devices are the target application of the battery system. SPEs are, however, typically generated in bulk by conventional radical thermal or UV-induced polymerization methods and then cast, dip- or spin-coated as thin films onto electrode materials prior to assembly in 2D battery configurations. ${ }^{5-13}$ A different, though far less common, approach is to generate thin polymer electrolyte (SPE) films directly onto the target electrode surface by in-situ methods. SPEs generated by this scenario provide a mechanism for applying these type of electrolytes onto architecturally complex 3D electrode structures, such as in small-scale high energy density batteries where the energy per footprint area can be significantly increased by two orders of magnitudes. ${ }^{14-16}$ Micro or nano scale batteries are essential for powering a range of autonomous microelectronic devices, such as medical implants and sensors. ${ }^{17,18}$ One of the difficulties in realising this concept is the need to coat complex non-planar electrode structures with a thin contiguous SPE film or, in the case of liquid electrolytes, apply the indispensable separator membrane. While prefabricated polymer films may be engineered into complex electrode arrangements, their mechanical handling and application can be very challenging, in particular if they are gel-like and/or strongly adhesive. Hence a convenient in-situ generation procedure is much sought after.

In this regard, electrochemical in-situ grafting or polymerization from monomer precursors is, compared to conventional radical thermal or UV-induced polymerization, very attractive for a number of reasons:

(i) the grafting process, polymer growth and SPE properties are easier to control since the self-limiting electrodeposition process can induce homogeneous stepwise conformal coatings over the entire electrode scaffold architecture resulting in blocking of the

zE-mail: muhammad.abdelhamid@csiro.au; Thomas.ruether@csiro.au substrate surface when the electrochemically generated polymer layer attains a certain thickness, ${ }^{18,19}$

(ii) ease of generation and application to the substrate electrode material, ${ }^{20}$

(iii) simplification of the battery fabrication process,

(iv) avoidance of polymer initiating reagents, and;

(v) grafting occurs directly at the target surface.

The last point is particularly important because it ensures intimate contact with the electrode surface which is critical for ion transport at the electrolyte-electrode interface and concomitant reduction in electrode resistances. This may be harder to achieve or not at all by bulk UV-initiated polymerization which, for example, is conducted with monomer material applied to the electrode surface.

Electrografting of polymers onto conducting surfaces has been demonstrated by Lecyon et al. in $1981^{19-23}$ and reviewed by Belanger et al. ${ }^{24}$ in 2011 as a highly effective method for surface modification of metals for corrosion protection, and by Voelcker et $\mathrm{al}^{25}$ for the modification of p-type silicon surfaces. However, this technique has only recently been applied to architecturally complex electrode materials in energy storage devices and only a few polymer electrolyte systems have been reported. Owen et al. described the coating of $\mathrm{MnO}_{2}, \mathrm{Ni}$ foam and carbon substrates with polyacrylonitrile (PAN) by electrochemically polymerising the monomer dissolved in acetonitrile ${ }^{26}$ or from the monomer saturated with oxygen. ${ }^{8}$ In these examples the materials obtained were subsequently soaked in a lithium salt solution to incorporate a lithium source into the polymer film. Di-Vona et al. reported the direct electrochemical grafting of a Li-ion conducting $p$-sulfonated poly(allyl phenyl ether) film onto $\mathrm{TiO}_{2}$ nanotubes from DMSO solution using cyclovoltammetric and chronoamperometric methods. ${ }^{27}$ Notably, Brandell et al. and Djenizian et al. have demonstrated the advantage of applying an electric potential to electrically conducting 3D electrode surfaces in contact with an acrylate monomer solution for the creation of SPE films on the surfaces in a controlled manner. Brandell and colleagues electrografted binary poly(propylene glycol)diacrylate/ lithium bis(trifluoromethanesulfonyl)imide (LiTFSI) films of nanometer thicknesses from an ethanolic monomer solution onto $\mathrm{Cu}_{2} \mathrm{O}$-coated $3 \mathrm{D} \mathrm{Cu}$ nano-pillars and estimated the ionic 
<smiles>[R]C(=C)C(=O)OC(C)(C)CCOC([R])(C)C</smiles><smiles>[R]C(C=O)=CC=[R]</smiles><smiles>[R]CCCCCCCC</smiles>

$\mathrm{A}=$<smiles>O=S(=O)(F)NS(=O)(=O)F</smiles>

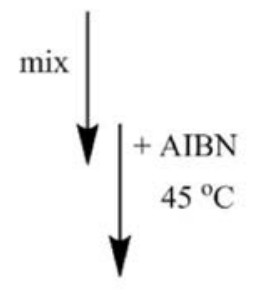<smiles>O=S(=O)(NS(=O)(=O)C(F)(F)F)C(F)(F)F</smiles>

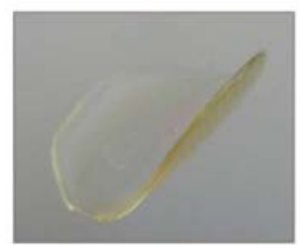

\section{Ternary polymer electrolyte}

Scheme 1. Formulation of monomer precursor mixtures and AIBN induced generation of SPE films.

conductivity of the resultant films to be in the order of $10^{-6} \mathrm{Scm}^{-1} \cdot{ }^{14,16}$ Djenizian et al. demonstrated the direct electrochemical polymerization of polyethylene oxide-b-methylmethacrylate co-monomer electrolyte from $0.035 \mathrm{M}$ aqueous LiTFSI solutions onto 3D nanostructured electrodes. ${ }^{28,29}$ It should be noted that most of the electrografted films were electrochemically polymerized from monomer solutions where radicals generated from the respective solvents (or from oxygen saturation $)^{26}$ initiated the polymerization as opposed to radicalization of the unsaturated functionality of the monomer reported by Jérôme et al. ${ }^{22}$

Herein, we present our approach for electrochemical grafting and polymerization of ultrathin polymer electrolytes (UTPE) onto 3D cylindrical micropillars and also onto planar disc electrodes from ternary monomeric electrolyte blends in the absence of sacrificial solvents. These blends consist of a poly(ethylene oxide)acrylate block co-monomer, a pyrrolidinium imide ionic liquid (IL) and a lithium imide salt. The resultant thin film polymers proved to be pinholefree and gave complete conformal coverage of the intricate 3Dstructured cylindrical electrodes. The morphology, composition and properties of the polymers produced were confirmed by differential scanning calorimetry (DSC), impedance spectroscopy, cyclic voltammetry, chronoamperometry, X-ray photoelectron spectroscopy (XPS), scanning electron microscopy (SEM) and energy-dispersive X-ray spectroscopy (EDS). Analysis of the quality of the thin films deposited provided insights on the feasibility and versatility of the electrochemical approach for creating continuous thin polymer films on complex electrically conducting surfaces.

\section{Experimental}

All solvents were analytical grade and used as received. The PEO-acrylate monomers were purchased from Sigma Aldrich and dried under high vacuum at ambient temperature prior to use in formulations. Azobisisobutyronitrile (AIBN, 98\%) and silver trifluormethylsulfonate $\left(\mathrm{AgCH}_{3} \mathrm{SO}_{3}\right.$, $\geq 99 \%$ ) were purchased from Sigma Aldrich. The ionic liquids 1-propyl-1-methylpyrrolidinium bis(fluorosulfonyl)imide (P13FSI, Wuhan Chemicals, China) and 1-butyl-1-methylpyrrolidinium bis(trifluoromethanesulfonyl)imide (P14TFSI, IoLiTec, Germany) were dried under high vacuum at $45 \pm 2{ }^{\circ} \mathrm{C}$ over 48 hours and subsequently stored against finely cut $\mathrm{Li}$ metal contained in a drying tube fitted to the storage flask. Lithium bis(fluorosulfonyl)imide, $\mathrm{LiN}\left(\mathrm{SO}_{2} \mathrm{~F}\right)_{2}$ (LiFSI, Boulder Ionics) and lithium bis(trifluoromethanesulfonyl)imide, $\mathrm{LiN}\left(\mathrm{SO}_{2} \mathrm{CF}_{3}\right)_{2}$ (LiTFSI, $3 \mathrm{M}$ ) were dried at $120^{\circ} \mathrm{C}$ under high vacuum for two days prior to use in formulations.

An n-type antimony-doped $15 \mathrm{~cm}$ diameter commercial $<111>$ silicon wafer (resistivity $=0.005-0.016 \mathrm{ohm} \mathrm{cm}$ ) of $360-390 \mu \mathrm{m}$ thickness (formerly WRS, now Pure Wafer, USA) was used as a substrate material for fabricating the cylindrical pillars upon which the UTPEs were deposited. The conductive silicon wafers were used as supplied. Deep reactive ion etching (DRIE) was used to generate high aspect ratio cylindrical pillars measuring $10 \mu \mathrm{m}$ in diameter and $100 \mu \mathrm{m}$ in height. The pillars were spaced $10 \mu \mathrm{m}$ apart. The dry etching process was carried out using an Oxford Instruments PLASMALAB100 ICP380 system. A mask purposely designed by CSIRO allowed 290 individual $\mu$-battery units (Figure S3) of varying dimensions (ranging from $3 \mathrm{~mm} \times 3 \mathrm{~mm}$ down to $100 \mu \mathrm{m} \times 100 \mu \mathrm{m}$ ) to be simultaneously produced on a single silicon wafer. A $40 \mathrm{~nm}$ conformal TiN layer was then deposited onto the silicon pillars via Atomic Layer Deposition (ALD) using Tetrakis(dimethylamido)titanium (TDMAT) as precursors with $\mathrm{Ar}, \mathrm{N}_{2}$, and $\mathrm{H}_{2}$ plasma at $250{ }^{\circ} \mathrm{C}$ to provide a strike layer for the subsequent $\mathrm{Ni}$ coating. A Cambridge Nanotech ALD Fiji F200 instrument was used to carry out the ALD process. The TiN-coated cylindrical pillars were subsequently electrochemically plated with a $1.2 \mu \mathrm{m}$ thick film of nickel which serves as a conducting layer. The DRIE, ALD and nickel electrodeposition work was carried out by the Melbourne Centre for Nanofabrication $(\mathrm{MCN})$.

The monomer blend solutions for the UTPEs were formulated according to Scheme 1 by mixing a commercially available pre-dried PEO-acrylate, a pyrrolidinium ionic liquid and a lithium imide in 
various proportions (10-40 wt\% IL rel. to monomer; $0.6-1.0 \mathrm{~m} \mathrm{Li}$ salt) inside an argon filled glove box.

Electrochemical polymerization and cyclic voltammetry were performed using an Autolab PGSTAT302N potentiostat (Metrohm) controlled by either the NOVA electrochemistry software or the GPES software. The electrochemical polymerization of the UTPE were carried out by applying a constant potential $(-1.75 \mathrm{~V}$ and $2.00 \mathrm{~V}$ vs. $\mathrm{Ag} / \mathrm{Ag}^{+}$) across the $3 \mathrm{D}$ electrode unit containing the cylindrical micropillar-electrodes or the planar 2D Ni, $\mathrm{Cu}$, or $\mathrm{Sn}$ disc electrodes. A Pt disc was used as the counter electrode and an $\mathrm{Ag}$ wire as the quasi reference electrode. A specially designed electrochemical cell (Figure S3) was fabricated to host the 3D micropillar substrates and the 2D planar disc electrodes to improve handling and ensure consistent experimental conditions between experiments. Another set of electropolymerization experiments were conducted using a standard three-electrode configuration cell in order to conduct cyclic voltammetry measurements on the deposited UTPE films. In this case, a gold (BAS surface area of $0.04 \mathrm{~cm}^{2}$ ) or a platinum (BAS, surface area of $0.04 \mathrm{~cm}^{2}$ ) electrode was used as the working electrode, $\mathrm{Ag} / \mathrm{AgCF} \mathrm{SO}_{3}$ (0.05 M in P14TFSI) as the reference electrode, and a Pt or stainless steel wire as the counter electrodes. Prior to each scan, the working electrode was first rinsed with deionized water, then coarse polished with $3 \mu \mathrm{m}$ alumina, followed by polishing with $0.3-1 \mu \mathrm{m}$ alumina, then rinsed with deionized water and HPLC grade methanol and finally dried.

The morphologies of the deposited UTPEs were imaged using a field emission scanning electron microscopy (Zeiss, Merlin, Jena, Germany). The elemental compositions of the UTPEs were determined by energy-dispersive X-ray spectroscopy (EDS) using an EDS detector and control software from EDAX.

Free standing polymer films for $T_{g}$ and complimentary ionic conductivity measurements were synthesized via conventional radical polymerization with AIBN initiator $(\sim 1 \mathrm{wt} \%)$ dispersed in the monomer blend at $45 \pm 5^{\circ} \mathrm{C}$. After curing overnight, free standing films of 0.5 to $1 \mathrm{~mm}$ thickness were readily obtained which are, however, difficult to detach from various surfaces, in particular glass, which makes it very tedious to mechanically handle thin and very sticky films. The problem could be alleviated to some extent by the use of Mylar or commercial silicone based baking moulds.

Differential scanning calorimetric (DSC) measurements were performed on a differential scanning calorimeter (TA DSC Q100). An average sample of $5 \mathrm{mg}$ was sealed in an aluminum pan and heated and cooled at a rate of $10^{\circ} \mathrm{C} \mathrm{min}-1$. Temperatures for the glass transition, crystallization and melting point were recorded.

Ionic conductivities were estimated by two different methods. One method involved using free standing films generated by bulk polymerization of the monomer initiated with AIBN. In the other method, electrochemically polymerized UTPE on flat 2D Ni electrodes were used in specially designed cells (Figure S4) as described below:

The ionic conductivities $(\sigma)$ of free standing films were measured by means of electrochemical impedance spectroscopy (EIS) in a coin cell type arrangement. The impedance tests were carried out over the frequency range from $0.1 \mathrm{~Hz}$ to $100 \mathrm{KHz}$ at an $\mathrm{AC}$ amplitude of $10 \mathrm{mV}$ using a potentio/galvanostat Solartron 1255B analyzer. A disc (15 mm diameter) was cut out of the polymer film, sandwiched between two stainless steel disks $\left(1.77 \mathrm{~cm}^{2}\right)$ supplied with the coin cell. The film thickness was estimated before and after the impedance measurement from this stack by subtracting the thickness of the two stainless steel discs. The measurements were conducted over a temperature range of $25-80^{\circ} \mathrm{C}$. The samples were thermally equilibrated for at least $12 \mathrm{~h}$ prior to each measurement at a selected temperature. The relative accuracy of all results was checked by replicating measurements under identical experimental conditions and by recording two data sets for each cell, a set of data collected during temperature ramp up and an equivalent data set for temperature ramp down; occasionally impedance data were also recorded at a certain temperature after the typical $12 \mathrm{~h}$ rest period and again after extended rest periods of 2-3 days at the same temperature; the retrieved data confirmed that a $12 \mathrm{~h}$ temperature rest time is sufficient for equilibration. In addition, the SPE film stack was recovered from coin cells after completion of measurements and the film thickness was estimated. From the collective of these observations the error margin of measurements for free standing films is $5-10 \%$. The ionic conductivities $(\sigma)$ of the polymer electrolytes were calculated based on the following equation:

$$
\sigma=\frac{d}{R_{r} A}
$$

where $d$ is the thickness of the polymer electrolyte film, $R_{r}$ is the ohmic resistance read from the real axis and $A$ is the area defined by the stainless steel electrodes holding the sample.

The ionic conductivity $(\sigma)$ of selected UTPE film created by electrochemical polymerization onto $2 \mathrm{D}$ substrates were measured by means of electrochemical impedance spectroscopy (EIS) similar to the freestanding films. However, given the UTPE's thinness, relative fragility and softness, sandwiching and pressing it between two electrodes might result in deformation and mechanical damage to the thin film. As a result, a drop of liquid metal alloy (Galinstan) was used as a soft electrode and the same special cell used for the electropolymerization on the $2 \mathrm{D} \mathrm{Ni}$ discs was used with a modified part to accommodate the liquid metal (Figure SX). The same parameters of EIS experiments on free standing polymer films were used.

$\mathrm{X}$-ray photoelectron spectroscopy (XPS) measurements were performed using a Kratos Axis Ultra DLD spectrometer. The instrument comprised of a monochromatic Al-K $\alpha(1486.6 \mathrm{eV}) \mathrm{X}$-ray source operating at $225 \mathrm{~W}$. The electron binding energy scale was calibrated for each sample by setting the C-C component of the $\mathrm{C} 1 \mathrm{~s}$ spectrum to $284.8 \mathrm{eV}$. XPS spectra were collected with pass energy of $160 \mathrm{eV}$ for the survey spectra and $40 \mathrm{eV}$ for the high-resolution spectra. Depth profiling was undertaken using an argon gas cluster ion source operating in cluster $\mathrm{Ar} 500^{+}$mode at $20 \mathrm{keV}$. A typical depth profile involved 100 survey/sputter cycles, with sputtering undertaken for 25 seconds per cycle. Data files were processed using CasaXPS software and interpreted using relative sensitivity factors provided by the instrument manufacturer (Kratos) as a guide. Background subtractions using a Shirley background were applied to all high-resolution spectra. Synchrotron XPS was conducted for measurements requiring the detection of lithium. These measurements were carried out at the Soft X-ray beamline of the Australian Synchrotron under ultra-high vacuum conditions with a base pressure of $5 \times 10^{-10}$ mbar or better. ${ }^{30}$ The tuneable energy range at the soft X-ray beamline of the Australian Synchrotron enables measurement at reduced photon energy $(100 \mathrm{eV})$ in order to achieve dramatic improvements in the sensitivity to lithium versus a lab-based instrument. A photon energy of $100 \mathrm{eV}$ was chosen due to its close proximity to the Li 1 s edge which significantly increases the photoionization cross-section resulting in approximately a 5000 fold increase in the counting statistics.

\section{Results and Discussion}

PEO-Acrylates were chosen for this application for three reasons. Firstly, the electrochemical reduction of standard acrylates (without a PEO moiety) is well established and their long term stability and effectiveness as coatings on metal surfaces has been demonstrated. ${ }^{19,23}$ Secondly, PEO-b-acrylate polymers have been shown to be promising matrixes for SPEs ${ }^{9,12,31}$ where the acrylate group with its polar oxygen constituent contributes to the ion transport. ${ }^{1}$ Thirdly, acrylate polymers are not only widely-accepted industrial compounds but they also have a low level of toxicity which renders them attractive electrolyte systems for biomedical applications such as implantable devices. ${ }^{20}$ In this work, we focused mainly on UTPEs based on poly(ethylene glycol) methyl ether acrylate (PEO-MA, $\mathrm{M}_{n} 480$ ). which typically gave higher conductivities reaching $5 \times 10^{-4} \mathrm{~S} \mathrm{~cm}^{-1}$ at $25^{\circ} \mathrm{C}$ compared to PEO-diacrylate counterparts, the latter having a less flexible network due to crosslinking via two acrylate groups present in the monomer. ${ }^{12}$ Similar ionic conductivities were reported for this type of ternary SPE by several other groups..$^{5,7,13,32}$ For the interested reader we present in the ESI temperature dependent conductivity studies as a function of (i) the nature of the acrylate - poly(ethylene glycol) methyl ether 


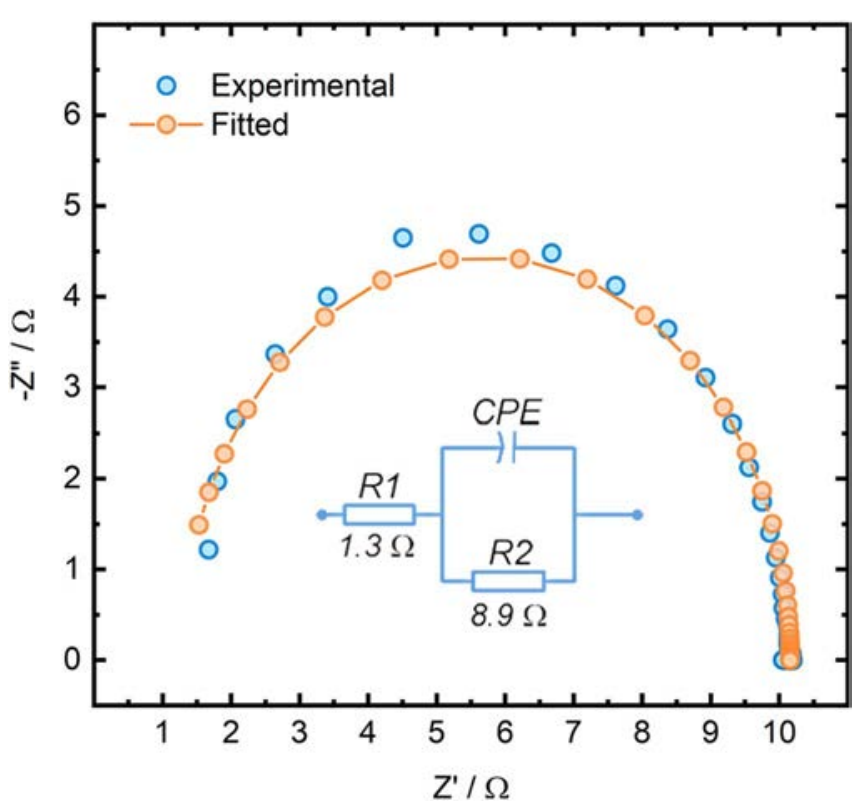

Figure 1. Nyquist plot obtained for an UTPE film of PEO-MA-30 created electrochemically on a 2D Ni surface.

methacrylate (PEO-MMA, $\mathrm{M}_{\mathrm{n}}$ 500), poly(ethylene oxide) diacrylate (PEO-DA, $M_{n}$ 575) and PEO- MA matrixes, respectively - (ii) as a function of the nature and (iii) the concentration of ionic components in the blend, along with a brief discussion of the data. Figure $1 \mathrm{~b}$ shows a Nynquist plot of experimental and fitted data obtained for an UTPE film of PEO-MA-30 created electrochemically on a 2D Ni surface.

Selected examples of SPE films were analyzed by DSC for glass transition temperature $\left(T_{g}\right)$, melting points and phase transitions (Table I). The majority of compositions examined display a flat profile with melting points above $120^{\circ} \mathrm{C}$. Ternary mixtures composed of two different ionic liquids and $0.6 \mathrm{~m}$ LiFSI show a crystallization event (exothermic peak) just before their melting. The lowest $T_{g},<$ $-50^{\circ} \mathrm{C}$, were observed for ternary mixtures where the ionic liquid and the lithium salt components are FSI salts, or if both FSI and TFSI anions are present in the polymer blend. Duluard and Delville et al. reported ${ }^{32}$ for ternary electrolytes BMIM-TFSI + Y wt $\%$ PMMA and a mol fraction of 0.03 LiTFSI, $\mathrm{T}_{\mathrm{g}}$ values of $-64^{\circ} \mathrm{C}(\mathrm{Y}=40 \%)$ and $0{ }^{\circ} \mathrm{C}(\mathrm{Y}=50 \%)$; Libo et al. who investigated similar SPE compositions did not disclose any DSC data. ${ }^{13}$ Based on our results from screening for conductivity and glass transition temperature, we selected the ternary composition PEO-MA $+30 \mathrm{wt} \%$ P13FSI $+0.6 \mathrm{~m}$ LiFSI (PEO-MA-30 in the following) for subsequent studies.

Electropolymerization.-Prior to conducting the electrochemical grafting experiments on the Ni-coated Si-based cylindrical 3D micropillar structured electrodes we investigated the electrochemical reduction of various acrylate monomer mixtures via cyclic voltammetry in a conventional three electrode cell. In the following our
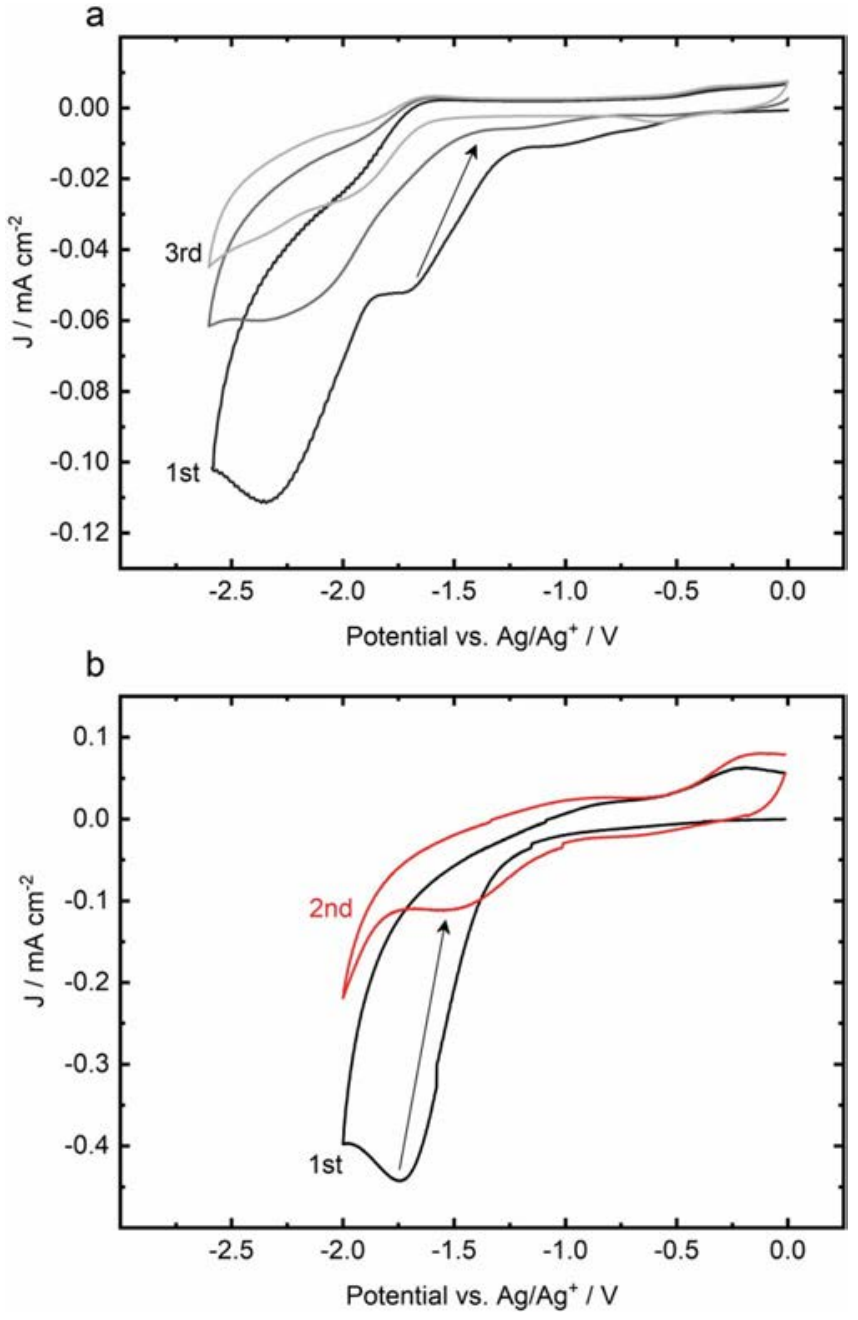

Figure 2. Cyclic voltammogram of PEO-MA-30 on a) Au electrode (2 mm diameter) and b) $\mathrm{Ni}$ disc (12 $\mathrm{mm}$ diameter) electrode at $20 \mathrm{mV} \mathrm{s}^{-1}$.

discussion will focus on one monomer blend only, results obtained for diacrylate based monomer formulations are summarized Figure S2 in the ESI. Figure 2a shows consecutive reductive scans of PEOMA-30 blend on a gold electrode. In the first cycle, the voltammetric response shows two irreversible reduction peaks at $-1.7 \mathrm{~V}$ and $-2.3 \mathrm{~V}$. The first reduction process corresponds to the grafting of the acrylate functional group onto the electrode surface whereas the second one is attributed to the bulk reduction of the acrylate functional group near the electrode surface and the growth of the polymer film. This is in agreement with previous observations by Jérôme et al. for acrylates and acrylonitriles dissolved in DMF solutions containing tetrabutylammonium hexafluorophosphate $\left(\mathrm{TBAPF}_{6}\right)$ supporting electrolytes. ${ }^{19,20}$ The first reduction process disappeared after the first

Table I. Thermal properties of selected ternary PEO-acrylate polymer electrolytes.

Formulation

PEO-MA + $20 w t \%$ P13FSI $+0.6 m$ Li-FSI

PEO-MA + $30 w t \%$ P13FSI + 0.6 m Li-FSI

PEO-MA + $30 w t \%$ P13FSI $+1.0 \mathrm{~m}$ LiTFSI

PEO-MA + $30 w t \%$ PI ITFSI + 0.6 m LiTFSI

PEO-DA + $30 w t \%$ P14TFSI + $0.6 \mathrm{~m} \mathrm{LiTFSI}$

PEO-MA + $30 w t \%$ P14TFSI:P13FSI $(1: 1)+0.6 \mathrm{~m} \mathrm{LiFSI}$

PEO-MA + $30 w t \%$ P14TFSI:P13FSI (1:1) + $0.6 \mathrm{~m}$ LiTFSI

PEO-MMA + $15 w t \%$ P13FSI + $0.6 m$ Li-FSI

$\mathrm{T}_{\mathrm{g}} /{ }^{\circ} \mathrm{C}$
-40.5
-53
-53.6
-45.4
-49
-59
-57
-38

m.p. $/{ }^{\circ} \mathrm{C}$

$>120$

$>120$

126.3

$>120$

139

115.7

120.5

$>142$
$\mathrm{T}_{\mathrm{C}} /{ }^{\circ} \mathrm{C}$

$-$

$-$



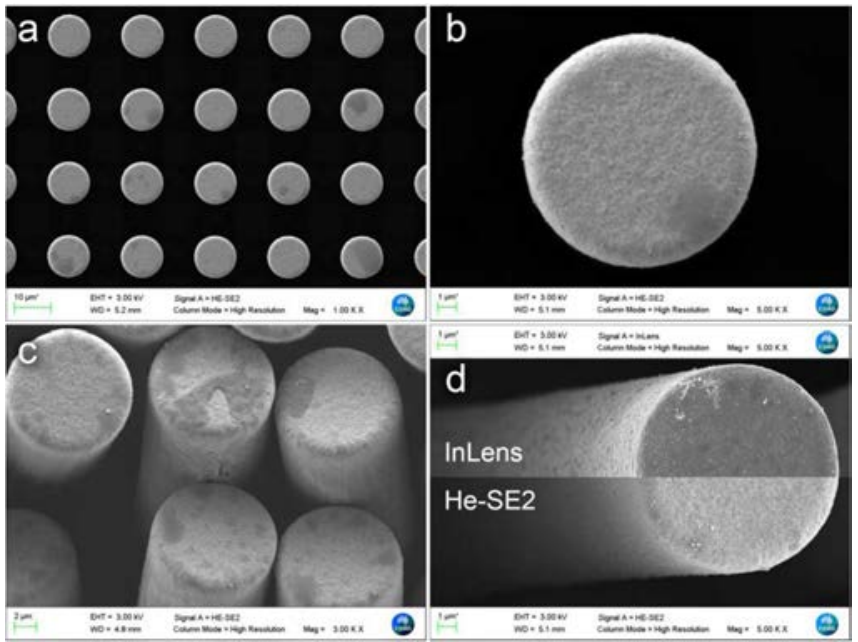

Figure 3. SEM images of 3D electrode scaffolds after electrografting of UTPE at $-1.7 \mathrm{~V}$ for $60 \mathrm{~s}$.

cycle which indicates the completion of the grafting process during the first cycle and the full functionalization of the electrode surface. In the subsequent cycles, the observed current density for the second reduction process rapidly decreased due to the insulating nature of the formed polymer. The self-limiting electrodeposition property of the process guarantees the complete coverage of the entire electrode surface with electronically insulating polymer electrolyte film and allows the control of its thickness by controlling charge density. ${ }^{18,19,27}$ Furthermore, when a more negative potential is applied an additional intense reduction process could be observed. ${ }^{24}$ Similar observations can be made for grafting processes using different substrates. Cyclic voltammetry measurements were similarly conducted on a $\mathrm{Ni}$ disc electrode in order to study the polymerization process on a surface similar to the Ni-coated 3D micropillar electrodes. In this instance, the potential window was limited to the first reduction potential in order to confine the study to the grafting process (ca. $-1.7 \mathrm{~V}$, Figure $2 \mathrm{~b})$. The voltammetric response on $\mathrm{Ni}$ is seen to be similar to that on the Au electrode. The current corresponding to the grafting process diminished significantly after the first cycle proving electrografting of the polymer film on the Ni surface took place.

After investigating the electrochemical polymerization process on planar 2D electrodes ( $\mathrm{Au}$, and $\mathrm{Ni}$ ) and establishing the optimum parameters and conditions, we conducted the same investigations on the Ni-coated 3D cylindrical micropillar electrode arrays present in the waver templates. However, constant potential deposition was employed using different deposition times $(60 \mathrm{~s}, 90 \mathrm{~s}$ and $120 \mathrm{~s})$ to obtain the polymer instead of cyclic voltammetry. To determine the optimum deposition potential value, two potential values were chosen (i.e. $-1.7 \mathrm{~V}$ and $-2.0 \mathrm{~V}$ ) which correspond to the first "grafting" and second "bulk polymerization" potentials, respectively, obtained from the studies on 2D electrodes. The UTPEs obtained on the intricate cylindrical 3D microelectrodes are shown in Figure 3. The electropolymerization process was first carried out for 60 s after which the electrodes were immediately washed with methanol to remove any loosely bound polymer and to stop further polymerization. The UTPE films produced at both potentials conformally coated the entire 3D electrode scaffold following the contour of the micropillars. The UTPEs grown at $-1.7 \mathrm{~V}$, i.e. grafting potential, show a consistent thickness across all regions of the 3D scaffold. On the other hand, at $-2.0 \mathrm{~V}$, i.e. the bulk polymerization potential, overgrowth of polymer was observed (Figure 4c) resulting in inter-pillar growth in the form of membranes or walls which is undesirable. Accordingly, the grafting potential of $-1.7 \mathrm{~V}$ was chosen to electrochemically polymerize UTPEs on the 3D electrodes as it allows for better control of the UTPE polymerization.

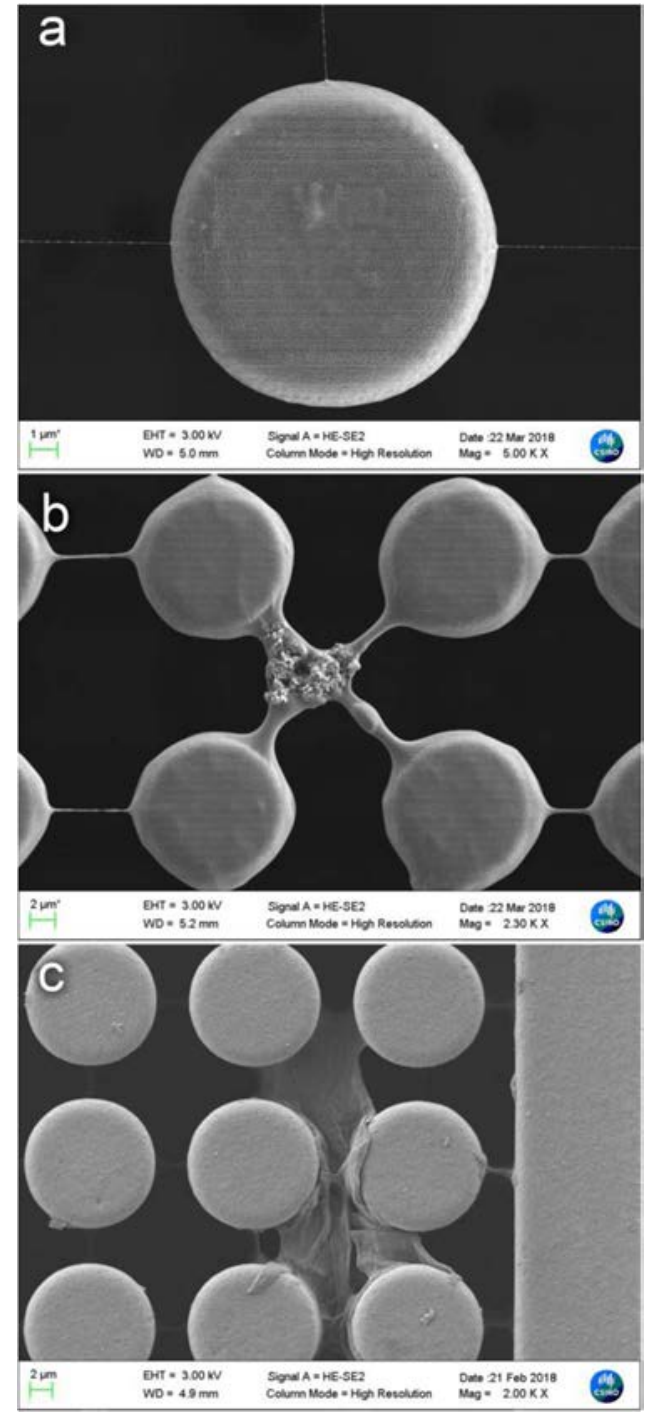

Figure 4. SEM images of 3D electrode scaffolds after UTPE was electrografted using different grafting times a) $90 \mathrm{~s}$, and b) $120 \mathrm{~s}$ at $-1.7 \mathrm{~V}$ and c) at $-2.0 \mathrm{~V}$ for $60 \mathrm{~s}$

The effect of longer polymerization times on the structure and morphology of the UTPEs was studied by applying the grafting potential for 90 s and 120 s (Figure 4). As expected, longer polymerization times resulted in thicker films around the pillars with no detectable variation in thickness. However, thicker films and overgrowths were produced at the top of the micropillars near the edge of the cylinders (Figure 4b). Polymer microstrings extending from the top of the micropillars to the neighboring micropillars were observed. The thickness and occurrence of these cross-linking microstrings increased with increasing polymerization time. The overgrown features are attributed to the higher current densities experienced at the top of the micropillars due to their geometry. ${ }^{33}$ The sharp edge at the top of the cylindrical shape of the pillar induces higher current density which is translated into thicker polymer films in these areas. This is in agreement with a recent study by Angelo et al. where they modelled the electrochemical and mechanical properties of trench geometry microbatteries. ${ }^{33}$

Furthermore, EDS elemental maps were recorded of the UTPEcoated micropillars in order to confirm the deposition of the polymer electrolyte (Figure 5). In particular, a damaged pillar was chosen for further examination as it allowed the coated pillar surface to be compared to the uncoated internal part of the micropillar. EDS data show a moderate silicon signal which is expected as the micropillars 

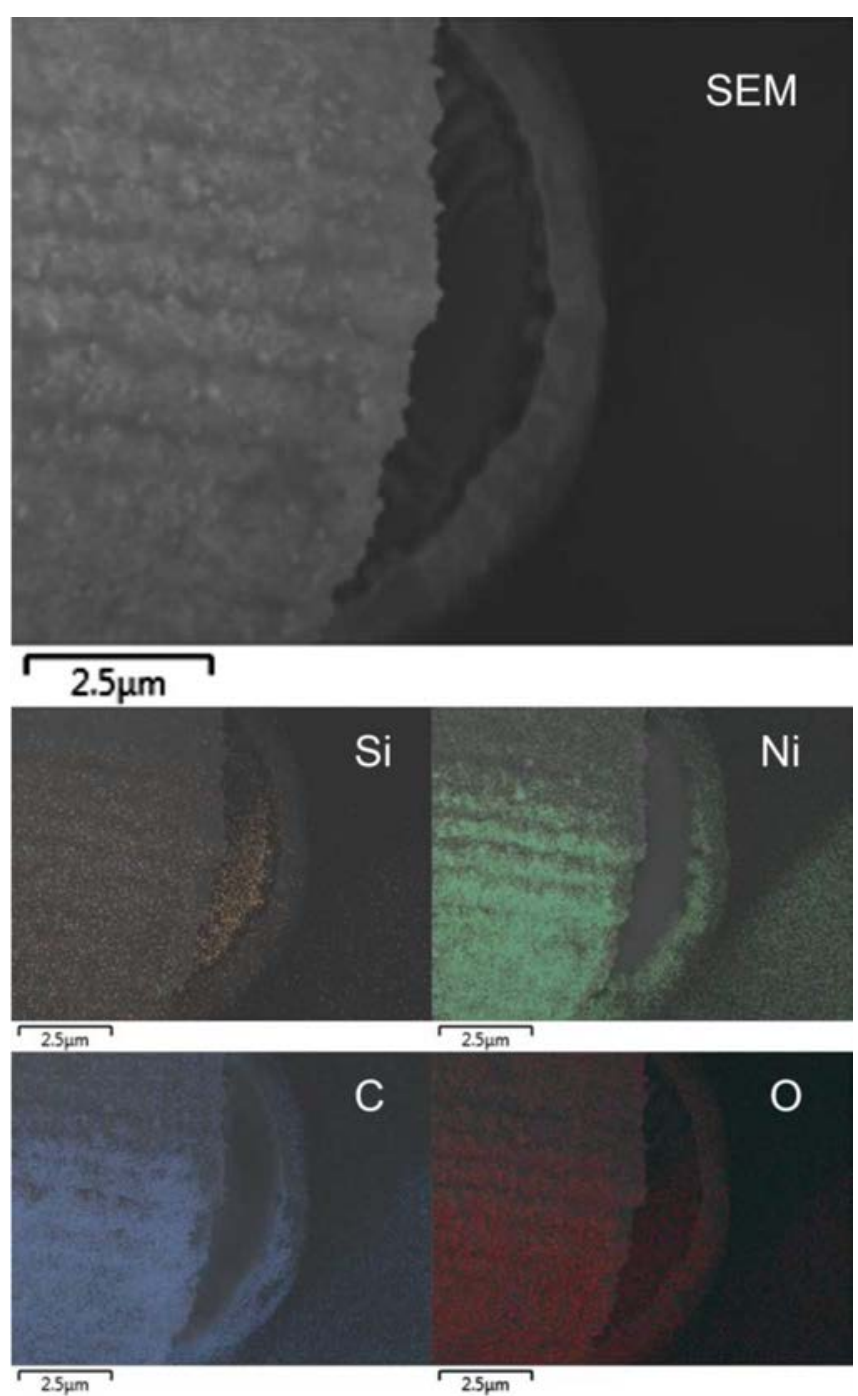

Figure 5. EDS elemental maps of the 3D electrode scaffolds after electrografting with the PEO-MA-30 UTPE.

are etched from a silicon wafer. The silicon signal is however lower on the sidewall of the pillar as it is masked by the nickel and polymer coating unlike the exposed internal section of the micropillar. The silicon signal can still be detected on the sidewall of the pillar due to the probing depth of the electron beam being longer than the thickness of the nickel and polymer layers combined. EDS also confirms the presence of nickel layer between the silicon and polymer. Carbon and oxygen, which are the main constituents of the polymer electrolyte film, can also be observed. The carbon mapped signal is similar to the nickel signal where it exists only on the surface of the pillar. The oxygen, on the other hand, is also mapped inside the collapsed pillar, which can be attributed to the oxygen from $\mathrm{SiO}_{2}$. The damage to the pillar happened outside the controlled environment of the glove box while transferring the sample to the SEM chamber.

To further complement and establish the composition of UTPE films, we examined XPS depth profiles of PEO-DA-based films obtained from different ternary monomer compositions using the electrochemical grafting method. Depth profile experiment showing survey spectra as a function of etch time of electrografted polymer electrolyte films on a copper surface are presented in Figures 6a, 6b. Figure $6 \mathrm{c}$ shows the relative atomic concentrations of each element (as calculated from the above survey spectra) as a function of depth. As expected, the polymer is relatively homogenous across the entire thickness of the electrochemical grafted polymer. A higher relative a

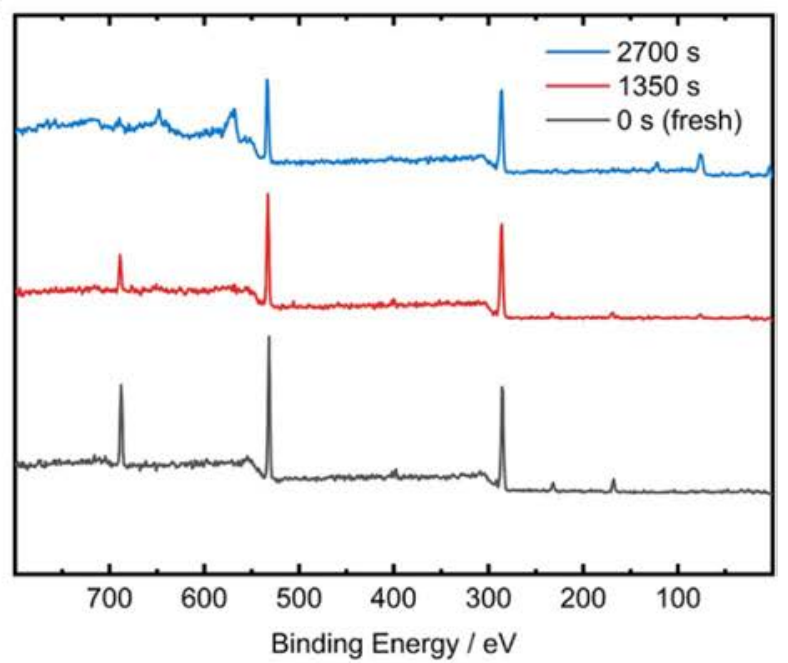

b

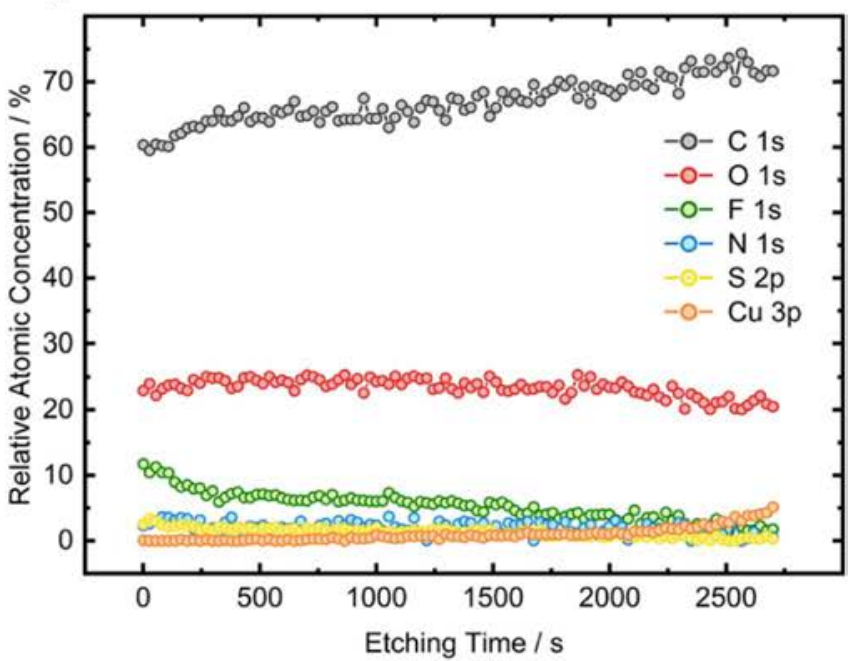

C

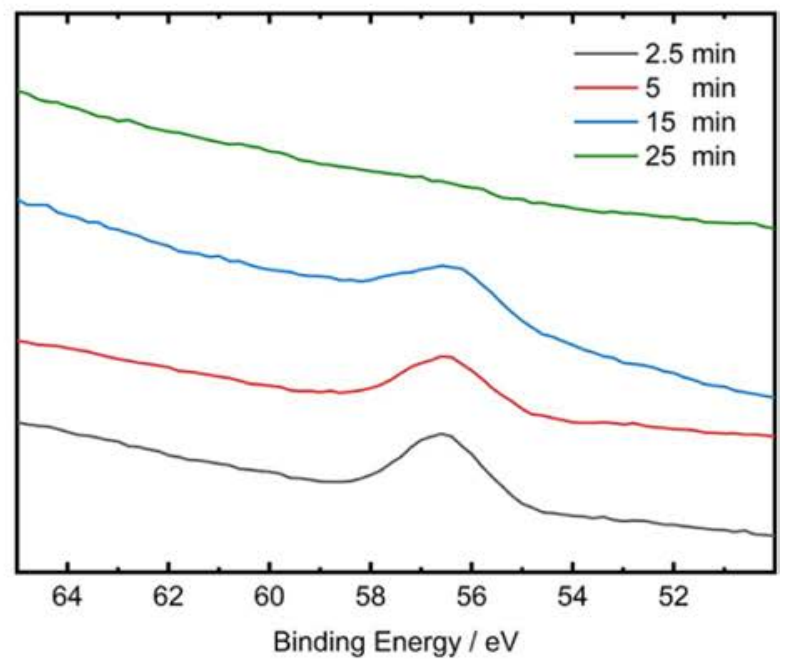

Figure 6. XPS depth profiling of the UTPE on copper substrate showing a) survey spectra collected at selected sputtering treatment times, b) the relative atomic concentrations of each element (as calculated from the survey spectra) as a function of depth, and c) the high resolution Li 1s signal. 
atomic concentration of fluorine and sulfur at the start of the depth profile may suggest a slight surface enrichment of the TFSI anion. Figure 6d presents the high resolution synchrotron-XPS Li 1s signal in the UTPE observed after different sputtering intervals. Notably, lithium is present throughout the entirety of the film up until 25 minutes of sputtering, coinciding with the appearance of $\mathrm{Sn} 3 \mathrm{~d}$ peaks associated with the tin substrate (i.e. the entire polymer film was sputtered away).

In subsequent experiments, we explored the feasibility of reversible lithium electrodeposition and striping from PEO-acrylate $+30 \mathrm{wt} \%$ P13FSI IL $+0.6 \mathrm{~m} \mathrm{Li}$-salt solution via cyclic voltammetry. Cyclic voltammograms at a Au disc working electrode are shown in Figure $7 \mathrm{a}$ for a still monomeric PEO-DA at a scan rate of $10 \mathrm{mV} \mathrm{s}^{-1}$ and $22^{\circ} \mathrm{C}$. The first scan shows a broad reduction processes at ca. -1.7 to $-2.5 \mathrm{~V}$ assigned to the electrografting processes as discussed earlier. The onset of lithium deposition begins at ca. $-3.3 \mathrm{~V}$ and shows a current crossover due to a nucleation and growth mechanism. The subsequent stripping of the deposited lithium metal occurs at ca. $-3.0 \mathrm{~V}$ with associated stripping processes at -2.3 and -2.0 (as shoulders) due to formation of $\mathrm{Li}-\mathrm{Au}$ alloy phases as expected during the plating process. The voltammetry demonstrates that on a Au electrode, the PEO-DA film does not fundamentally change the chemically reversible lithium plating stripping reaction. The electrochemical data does not show electrochemical reversibility, as expected, due to the concomitant solid electrolyte interphase formation due to ionic liquid breakdown $\mathrm{n}^{34}$ and subsequent lithium salt formation on the deposited lithium close to the deposition potential. Similar behavior is observed at a Pt working electrode where the first scan shows the electrografting processes (Figure $7 \mathrm{~b}$ ) at ca. $-1.6 \mathrm{~V}$ and $-2.2 \mathrm{~V}$. Lithium plating begins at $-3.5 \mathrm{~V}$ and again shows the current crossover due to nucleation and growth mechanism. In contrast to the Au working electrode, only a single oxidation peak at $-2.6 \mathrm{~V}$ due to lithium stripping is observed due to formation of Li metal only upon plating. Cyclic voltammograms at a $\mathrm{Ni}$ disc electrode (Figure 7c) shows chemically reversible lithium deposition and stripping across the PEO-MA-30 UTPE at a scan rate of $20 \mathrm{mV} \mathrm{s}^{-1}$. The voltammetry again shows nucleation loops (current crossover) demonstrating the lithium being electrodeposited in the expected nucleation and growth mechanism. Similar to the $\mathrm{Pt}$ electrode case, only a single stripping process due to lithium plating is observed. In the case of the Ni electrode, additional processes due to incomplete electrografting can be observed.

Overall the voltammetric study demonstrates lithium plating and stripping can be achieved in the presence of the electrografted polymer electrolyte films. These films do not fundamentally affect the nature of the lithium plating and stripping reaction, and for all three electrode substrates utilized in this study, the voltammetry showed the as-expected responses. This would then suggest that the electrografted polymer electrolyte films may have the potential for use in a li-ion battery. Further optimization and studies will focus on understanding if this hypothesis can be validated.

\section{Summary}

In this work we have demonstrated that the surface of architecturally complex electrode structures, i.e. 3D micropillars, can be conveniently coated with UTPE films electrochemically from multicomponent monomer mixtures. The created films consisting of PEOacrylate $+\geq 30 \mathrm{wt} \%$ P13FSI $+\geq 0.6 \mathrm{~m} \mathrm{LiFSI}$ have a room temperature ionic conductivity $>10^{-4} \mathrm{~S} \mathrm{~cm}^{-1}$. The electrografting method adopted here is very attractive because it allows control over the UTPE film formation by adjusting the polymerization potential and time. The presented electrografting process which incorporates the lithium salt in situ without the need for any further processing steps is also attractive because it creates an intimate contact between the conducting electrode surface and the UTPE film which is essential for effective ion-transport during electrochemical operations. Thus, cyclic voltammetric experiments conducted on these films showed reversible lithium deposition and stripping which provides further evidence for the $\mathrm{Li}^{+}$transport across the UTPE produced by our
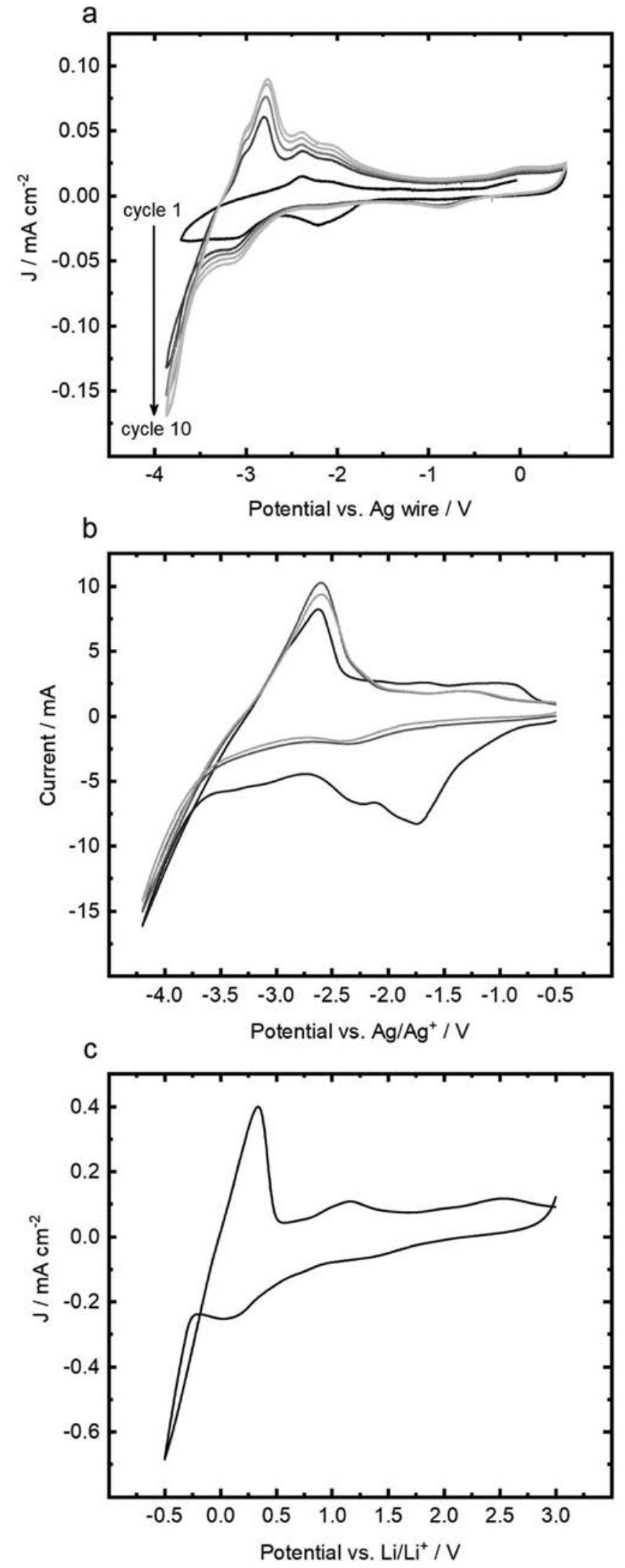

Figure 7. (a) Cyclic voltammogram of monomer mixture PEO-DA $+30 \mathrm{wt} \%$ $\mathrm{C}_{3}$ mpyr-FSI + $0.6 \mathrm{~m}$ LiTFSI; WE: Au, RE: Ag/AgOTf, CE: Pt; $\nu=10 \mathrm{mVs}^{-1}$ (b) Cyclic voltammogram of monomer mixture PEO-MA + $30 \mathrm{wt} \% \mathrm{P} 13 \mathrm{FSI}+$ $0.6 \mathrm{~m}$ LiTFSI; WE: Pt foil, RE: $\mathrm{Ag} / \mathrm{AgCH}_{3} \mathrm{SO}_{3}$, CE: Pt; $v=50 \mathrm{mV} \mathrm{s}^{-1}$ and (c) Cyclic voltammogram of P13FSI $+0.6 \mathrm{~m}$ LiFSI obtained on a Ni electrode coated with PEO-MA-30 polymer film; RE: $\mathrm{Li}, \mathrm{CE}: \mathrm{Li}, \nu=20 \mathrm{mV} \mathrm{s}^{-1}$. 
methodology. These developments are very encouraging for future miniaturized solid state Li-ion battery developments.

\section{Acknowledgments}

The authors acknowledge the facilities, and the scientific and technical assistance of the WA X-Ray Surface Analysis Facility, funded by an Australian Research Council LIEF grant (LE120100026). The authors are grateful for the technical support provided by B. Cowie and A. Tadich during synchrotron-XPS measurements at the Australian Synchrotron. The authors also thank Maja A. Schuknecht for conducting a series of conductivity measurements as part of an internship at CSIRO and the team of Bernie Orelup of the Melbourne Centre for Nanofabrication (MCN) for helpful discussions relating to the fabrication of 3D microelectrode substrates.

\section{ORCID}

Muhammad E. Abdelhamid (D)

https://orcid.org/0000-0002-1666-6398

\section{References}

1. J. Kalhoff, G. G. Eshetu, D. Bresser, and S. Passerini, ChemSusChem, 8, 2154 (2015) 2. A. S. Shaplov et al., Polym. Sci. Ser. B, 56, 164 (2014).

3. Zhengcheng Zhang et al., (2003) https://pubs.acs.org/doi/abs/10.1021/ma0349276.

4. M. B. Armand and M. Duclot, (1981) https://patents.google.com/patent US4303748/en.

5. A. S. Lee et al., Electrochimica Acta, 215, 36 (2016).

6. S. D. Tillmann, P. Isken, and A. Lex-Balducci, Journal of Power Sources, 271, 239 (2014).

7. M. Tosoni, M. Schulz, and T. Hanemann, Int. J. Electrochem. Sci., 9, 16 (2014).

8. M. J. Lacey, M. Sosna, and J. R. Owen, Electrochimica Acta, 96, 23 (2013).
9. L. Wang, N. Li, X. He, C. Wan, and C. Jiang, J. Electrochem Soc, 159, A915 (2012)

10. S. Tan, S. Walus, T. Gustafsson, and D. Brandell, Solid State Ionics, 198, 26 (2011).

11. B. Sun, I.-Y. Liao, S. Tan, T. Bowden, and D. Brandell, Journal of Power Sources, 238, 435 (2013)

12. M. Morita, M. Ishikawa, and K. Asanomi, Solid State Ionics,113-115, 117 (1998)

13. L. Libo, L. Jiesi, Y. Shuo, G. Shaowen, and Y. Peixia, Colloids and Surfaces A. Physicochemical and Engineering Aspects, 459, 136 (2014).

14. M. Valvo et al., J. Mater. Chem. A, 1, 9281 (2013).

15. K. Edström, D. Brandell, T. Gustafsson, and L. Nyholm, Electrochem. Soc. Interface, 20, 41 (2011).

16. B. Sun, D. Rehnlund, M. J. Lacey, and D. Brandell, Electrochimica Acta, 137, 320 (2014).

17. J. W. Long, B. Dunn, D. R. Rolison, and H. S. White, Chem. Rev., 104, 4463 (2004)

18. J. F. M. Oudenhoven, L. Baggetto, and P. H. L. Notten, Advanced Energy Materials, 1, $10(2011)$

19. S. Gabriel et al., Angewandte Chemie International Edition, 44, 5505 (2005).

20. S. Gabriel, R. Jérôme, and C. Jérôme, Progress in Polymer Science, 35, 113 (2010).

21. G. Lecayon, G. C. Le, and M. A. Le, (1981) https://patents.google.com/ patent/EP0038244A1/en?oq=0038244.

22. N. Baute, L. Martinot, and R. Jérôme, Journal of Electroanalytical Chemistry, 472 83 (1999).

23. N. Baute et al., European Journal of Inorganic Chemistry, 2001, 1097 (2001)

24. D. Bélanger and J. Pinson, Chem. Soc. Rev., 40, 3995 (2011).

25. D. Losic, M. Cole, H. Thissen, and N. H. Voelcker, Surface Science, 584, 245 (2005).

26. G. El-Enany, M. J. Lacey, P. A. Johns, and J. R. Owen, Electrochemistry Communications, 11, 2320 (2009).

27. I. V. Ferrari, M. Braglia, T. Djenizian, P. Knauth, and M. L. Di Vona, Journal of Power Sources, 353, 95 (2017).

28. N. A. Kyeremateng, F. Dumur, P. Knauth, and T. Djenizian, ECS Trans., 33, 77 (2011).

29. N. Plylahan et al., Nanoscale Research Letters, 7, 349 (2012).

30. B. C. C. Cowie, A. Tadich, and L. Thomsen, AIP Conference Proceedings, 1234, 307 (2010)

31. X.-X. Zeng et al., J. Am. Chem. Soc., 138, 15825 (2016)

32. S. Duluard et al., Electrochimica Acta, 55, 8839 (2010).

33. D. Grazioli, V. Zadin, D. Brandell, and A. Simone, Electrochimica Acta (2018) http://www.sciencedirect.com/science/article/pii/S0013468618316608.

34. A. Basile, A. I. Bhatt, and A. P. O'Mullane, Nature Communications, 7 , ncomms11794 (2016). 